\title{
Personalized Virtual Reality for Upper Extremity Rehabilitation: Moving from the Clinic to a Home Exercise Program
}

\author{
Cherie Behar ${ }^{1}$, Maxwell Lustick ${ }^{2}$, Matthew H. Foreman², Jennifer Webb² and \\ Jack R. Engsberg, ${ }^{2}$ \\ ${ }^{1} 2342$ Springside Drive, Colorado Springs, CO 80951, USA \\ ${ }^{2} 4444$ Forest Park Ave., CB 8505, St. Louis, MO 63108-2212, USA
}

\begin{abstract}
Introduction: Traditional rehabilitation does not provide adequate repetitions for maximal motor recovery in the clinic and home exercise programs (HEPs) have low compliance rates. Personalized virtual reality (PVR) is a promising low-cost therapeutic tool for improving compliance by incorporating the client's interests, abilities, and goals into a motivating and engaging intervention using internet games.

Objectives: The current study aimed to develop and refine a clinic-to-home PVR intervention, determine its feasibility and usability in an outpatient rehabilitation clinic and as a HEP, and examine its effects on motivation/engagement,
\end{abstract} compliance, motor repetitions, and functional motor performance.

Methods: The PVR system utilizes a Microsoft Kinect sensor to track the participants' movements, free software to translate movements to keystrokes, and free internet games. The therapist matched participants' interests to internet games, customized therapeutic movements for game play, and increased the movement thresholds for game activation as participants improved.

Two participants who had strokes resulting in upper extremity (UE) hemiplegia were recruited. The participants attended outpatient occupational therapy (OT) services twice weekly. Following training, the participants used the PVR system at home in place of their UE HEP. They continued to receive traditional OT once a week and clinic-PVR once a week for 58 weeks.

Results: The PVR intervention was successfully implemented in the clinic and the clients' homes. PVR increased motivation and treatment compliance. The clients exhibited improvements in UE active range of motion, function, symptoms, and occupational performance.

Conclusion: Preliminary evidence suggests PVR can improve motivation, compliance, function, and occupational performance. However, larger scale studies and protocol refinement are necessary.

Keywords: Occupational therapy, client-centered, stroke, hemiparesis, motivation, occupational performance, home exercise program, upper extremity rehabilitation.

Stroke affects 6.8 million people and is the leading cause of long-term disability in the United States [1]. Traditional stroke rehabilitation typically involves numerous outpatient rehabilitation visits and completion of a home exercise program (HEP). However, clients only complete about eight percent of the approximately 10,000 challenging repetitive movements required to maximize motor recovery in the clinic [2] and only one-third of clients complete their HEP as prescribed [3]. This is due to the tedious and non-motivating nature of therapeutic activities $[3,4]$.

Stroke rehabilitation research has shown that virtual reality interventions can provide a substantial number of task-oriented repetitions at a high-intensity, resulting in improved motor performance [5] that generalizes to real-world tasks [6]. These results are likely attributable to the motivating and engaging format of virtual reality [7-9].

*Address correspondence to this author at 4444 Forest Park Ave., CB 8505 , St. Louis, MO 63108-2212, USA; Tel: 314-286-1632; Fax: 314-286-1601; E-mail: engsbergj@wustl.edu
Personalized Virtual Reality (PVR) may further increase motor recovery by improving compliance and motivation through customized games to meet clients' interests, abilities, and goals. Clients have been shown to complete $300-600$ movements during a 40 -minute PVR session $[10,11]$, that is $3-6$ percent of the total repetitions needed in a single session. PVR has also improved quality-of-life and participation in daily activities for persons with chronic stroke and cerebral palsy. Furthermore, PVR is low-cost, portable, easy-touse and set-up, can be used with children and adults, and in a variety of settings [9-11].

The purpose of this study was to develop and refine a clinic-to-home PVR intervention and determine its feasibility and usability in an outpatient rehabilitation clinic and as a HEP. It was hypothesized that PVR would be feasible in both the clinic and home and would improve compliance and functional motor performance by providing individuals with a motivating and engaging experience. 


\section{INTERVENTION}

\section{Participants}

Two participants were recruited through an outpatient occupational therapist (OT) at a rehabilitation hospital. Participant 1, a 57-year-old African-American male, had experienced two strokes. His first stroke, four years prior to the intervention, resulted in right-sided hemiparesis and a second stroke, one year prior to the intervention, resulted in left-sided hemiparesis. Prior to his second stroke, Participant 1 was left-hand dominant and independent with all of his activities of daily living (ADLs). At baseline, he used his right arm for all tasks and required assistance with most ADLs. Participant 2, a 50-year-old African-American male, experienced a stroke resulting in right-sided hemiparesis and significant spasticity in his right upper extremity (UE). He was right-hand dominant and independent in all of his ADLs prior to his stroke. The Institutional Review Boards at Washington University School of Medicine and the rehabilitation hospital approved the investigation. Written informed consent was obtained prior to study commencement.

\section{Hardware and Software}

Equipment required to play PVR games included a Microsoft Kinect sensor, laptop, USB cable, high-speed internet, and an optional TV or monitor for larger viewing. The Kinect sensor was used with free Flexible Action Articulated Skeleton Toolkit (FAAST) software to translate UE movements into keyboard strokes for controlling internet-based PVR games. The Kinect sensor is an inexpensive $(\sim 990)$ motion sensing accessory to the Microsoft Xbox that uses a color camera, depth sensor, and algorithms to form a 3dimentional skeleton of a moving person in real time (Figure 1). The Xbox console and Xbox games were not used as the Kinect plugs directly into the laptop where the participants can play free internet games.

The FAAST software converts gross body movements (input) into keyboard strokes or mouse movements (output) (http://youtu.be/eM2cAMgMbzU). The ability to set thresholds to translate body movements into game movements permits thousands of internet games to become personalized interventions. These games are found by searching the internet for games that correspond with a participant's interests such as puzzles, baseball, pinball, or racing. MATLAB software was also used to create a "launch pad" that automatically loaded the recording program, internet games, and FAAST for simplification of computer navigation. Due to the large amount of data being collected, date, time, quality, and number of repetitions performed during game play data were stored directly on the laptop's hard drive. Game links

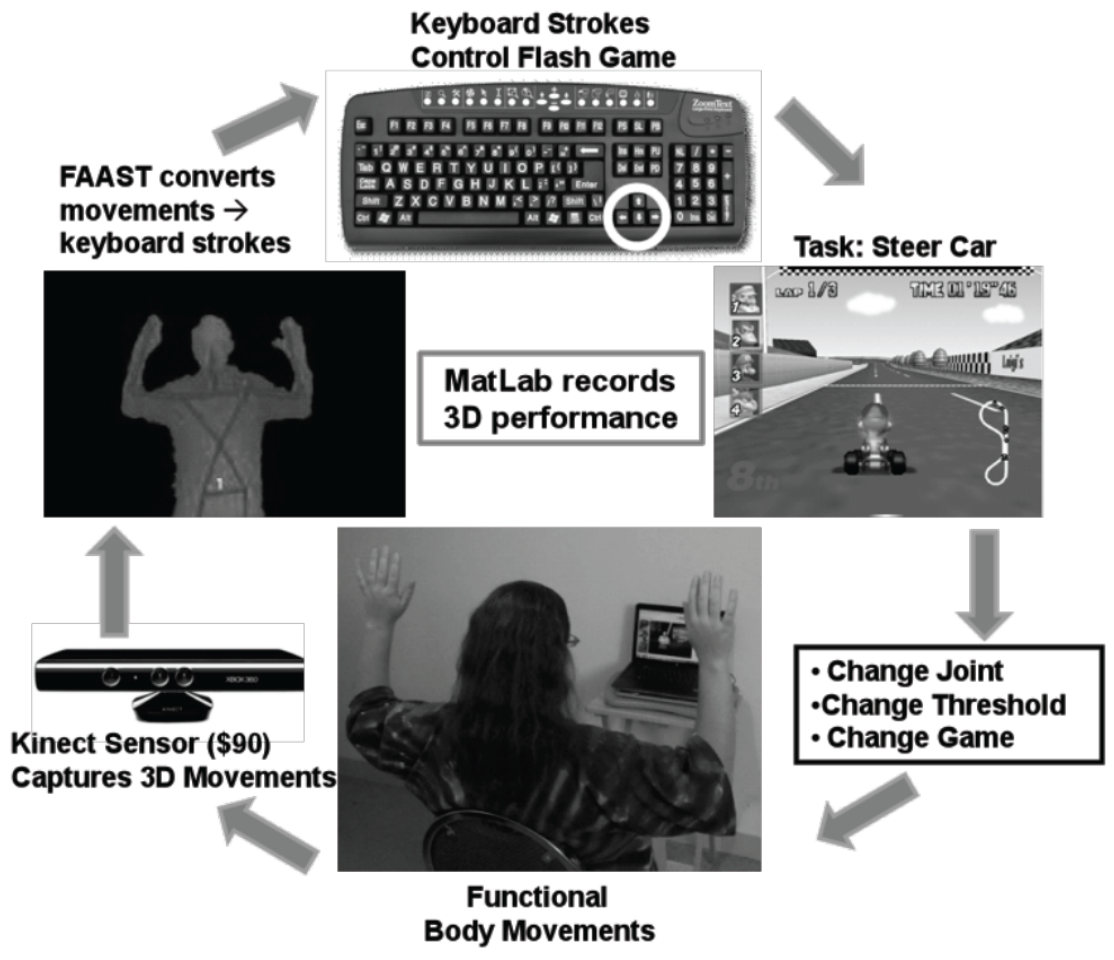

Figure 1: PVR system. 
and FAAST configuration files were stored on a cloud server, allowing therapists and researchers to update games without the participant needing to bring equipment to appointments.

\section{PVR Protocol and Design}

During the intervention, both participants attended outpatient OT services twice weekly to address functional limitations. One session per week was designated for traditional OT activities including education (e.g., energy conservation strategies) and practicing strategies for performing ADLs (e.g., dressing, bathing, eating) and instrumental ADLs (e.g., cooking, shopping, housework). The second weekly session was for clinic-PVR and took the place of usual UE exercises, which were used as preparatory activities for functional tasks.

Following initial assessments, both participants were scheduled for four consecutive clinic-PVR sessions to learn how to independently set-up and fully utilize the system in their home. Following training, clinic-PVR occurred once per week for 30 to 45 minutes and the participants were loaned the PVR system to take home and use in place of their previous UE HEP.

In order to improve multiple functional movements in various affected UE joints, PVR sessions consisted of playing three different games for 10-20 minutes each. Each game required different movements to activate the game controls. For example, Game 1 may address elbow flexion, Game 2 shoulder external rotation, and Game 3 shoulder flexion. As participants improved, the degree of movement required to activate the game controls was increased to maintain a sufficient level of challenge. After 5-8 weeks, the participants returned the system and were reassessed.

Due to the client-centered nature of the study, some aspects of the two participants' experiences differed. This included differences in scheduling rehabilitation sessions and their individual PVR progression schedules. Participant 1 began the HEP three weeks into the study with no previous PVR experiences. However, due to schedule differences and technical difficulties, Participant 2 received clinic-PVR inconsistently for three months before training to use the system independently and taking the system home. The participants' prescribed HEP schedule was also different. Participant 1 was instructed to follow a schedule that gradually increased the number of days played each week in order to reach the minimum of 9,600 challenging repetitions needed for maximal motor recovery [2] within eight weeks (Table 1). Participant 2 was encouraged to start with two 30 to 45 minute sessions per week and play as often as tolerated. Lastly, the timing of the assessments varied between the participants due to individual schedules and requirements of the rehabilitation therapist.

\section{ASSESSMENT MEASURES}

\section{Key Informant Interviews}

Semi-structured interviews were conducted at study completion. Participant interviews focused on gaining their perspectives on PVR's effectiveness and the system's usability. The outpatient OT delivering the interventions was also interviewed using open-ended questions regarding feasibility, usability, usefulness,

Table 1: PVR Progression Schedule for Participant 1

\begin{tabular}{|c|c|c|c|c|c|}
\hline Study Week & $\begin{array}{l}\text { 30-Minute Clinic } \\
\text { PVR Sessions }\end{array}$ & $\begin{array}{l}\text { Clinic UE Motor } \\
\text { Repetitions }\end{array}$ & $\begin{array}{l}\text { 60-Minute Home } \\
\text { PVR Sessions }\end{array}$ & $\begin{array}{l}\text { Home UE Motor } \\
\text { Repetitions }\end{array}$ & $\begin{array}{l}\text { Total UE Motor } \\
\text { Repetitions }\end{array}$ \\
\hline Week 1 & 1 & 143 & 0 & 0 & 143 \\
\hline Week 2 & 2 & 286 & 1 & 306 & 592 \\
\hline Week 3 & 2 & 286 & 2 & 612 & 898 \\
\hline Week 4 & 1 & 143 & 4 & 1224 & 1367 \\
\hline Week 5 & 1 & 143 & 5 & 1530 & 1673 \\
\hline Week 6 & 1 & 143 & 5 & 1530 & 1673 \\
\hline Week 7 & 1 & 143 & 5 & 1530 & 1673 \\
\hline Week 8 & 1 & 143 & 5 & 1530 & 1673 \\
\hline Total & 10 & 1430 & 27 & 8262 & 9692 \\
\hline
\end{tabular}

Note: PVR = Personalized Virtural Reality; UE = Upper Extremity. 
and implementation challenges. The caregiver of Participant 1 was interviewed to capture her perspective on the changes in his functional abilities.

\section{Compliance and Repetitions}

Compliance and number of repetitions were collected using MATLAB, which automatically recorded date, time, duration of play, and body coordinates for determining the quality and quantity of movement repetitions.

\section{Passive and Active Range of Motion (ROM)}

ROM measurements followed standard procedures using a goniometer. Measurement areas were personalized based on each participant's UE rehabilitation focus. Active ROM can be used to track differences in a person's ability to independently perform specific movements indicating functional capacity. Passive ROM measures the movement of joints manually by the therapist. Improvements in passive ROM often precede active ROM. The use of goniometers is accepted as a valid clinical tool for collecting ROM [12]. Overall, ROM assessment has shown better intrarater than interrater reliability. To ensure reliability, the same person completed the ROM measurements.

\section{Motivation and Engagement}

Motivation and engagement were assessed every two weeks for Participant 1 and twice for Participant 2. The interest/enjoyment and effort/importance subscales of the Intrinsic Motivation Inventory (IMI) were used in addition to the participants' continued feedback. The IMI uses a series of positive and negative statements to which the participant responds with a Likert-style answer in how well he agrees with the statement. The IMI has been shown to reflect the overall level of intrinsic motivation for an activity and the interest/ enjoyment subscale has good internal reliability [13].

\section{Action Research Arm Test (ARAT)}

The ARAT is a standardized assessment of UE function. The protocol has four subscales including grasp, grip, pinch, and gross movement. It was delivered and scored by the therapist. The range of scores is from 0 (severe disability) to 57 (no observed disability). On average, clients with chronic stroke score 29.2 on the ARAT with a standard deviation of 12.5. The ARAT has a minimally clinically important difference (MCID) of 5.7 [14].
The Disabilities of the Arm, Shoulder, and Hand (DASH)

The DASH is a self-report questionnaire that was given every two weeks throughout the course of the study and used to measure perceived UE function and symptoms. DASH total scores range from zero (no disability) to 100 (most severe disability) with a 10-point change being considered clinically important [15]. The $\mathrm{DASH}$ can also measure changes over time or with treatment [16]. The DASH has excellent test-retest reliability $\left(r=0.96 ; \quad r_{s}=0.95\right)$ and good discriminant validity.

\section{Canadian Occupational Performance Measure (COPM)}

Occupational performance was measured at baseline, onset of home PVR, and post-intervention using the COPM. The COPM assesses perceived level of importance, satisfaction, and performance of activities in the areas of self-care, productivity, and leisure. Items are rated on a scale of one to 10 with one being low and 10 being high. Total performance and satisfaction scores can also be calculated using the average ratings of all activities rated [17]. A twopoint increase in satisfaction and/or performance is considered a clinically important change [18].

\section{RESULTS}

\section{Weekly Feedback}

During the intervention, weekly feedback from the participants and therapist was used to refine the PVR games and protocol. For example, some games initially chosen to coincide with the participants' interests, were replaced after negative feedback in the clinic. Reasons for changing the games included frustration due to the level of difficulty (e.g., the speed at which the game was required to be played) and visual appeal. ClinicPVR visits were also used to troubleshoot any technical issues and change games and activation thresholds.

\section{Key Therapist Findings}

The therapist noted higher levels of client engagement, motivation, and compliance, and increased use of the affected limbs. She believed that both participants completed the home-PVR more than their previous HEPs. Since neither participant used their affected arms for functional activities prior to the PVR intervention, she thought the repetitions completed during PVR were extremely important to 
recovery. She also noted several challenges that are discussed in limitations. The therapist recommended the following based on her experience with this pilot study when considering clients for future PVR interventions:

Clients most likely to benefit:

- $\quad$ Orthopedic (localized impairments within the UE)

Clients least likely to benefit:

- Moderate-severe cognitive deficits (e.g., current cognitive goals)

- $\quad$ Serious medical comorbidities (e.g., renal failure)

- Hypertonicity (e.g., many games require fast reactions)

\section{Key Findings from Participants and Caregiver}

Participant 1's caregiver completed set-up and troubleshooting of minor problems easily using the provided manual. The participant could play games independently once the launch pad was opened. He reported improved compliance when compared to his previous HEP. He attributed this to the excitement of the games, trying to improve scores, and having a variety of games to avoid boredom. However, the participant reported that he was unable to play games according to the protocol due to fatigue and illness caused by his comorbidities. The participant also reported experiencing fatigue for 30 to 45 minutes after playing, but that this did not affect his functional activities. He also believed PVR was beneficial for getting in more repetitions. The participant noticed he could reach higher with his left arm and that he was using his left arm more often and without conscious thought for functional activities including writing, feeding, and brushing his teeth.
Participant 2 reported minor technical difficulties using the system at home. These were solved by a family member or during the next clinic-PVR session. $\mathrm{He}$ said that the system was fun and easy-to-use. He would enthusiastically recommend the system to others in a similar situation. Participant 2 reported using PVR four times per week in 40-minute sessions. He experienced no soreness or pain after sessions because it was not perceived to be as strenuous as his other, full-body HEP, but he felt the process was strengthening his arm.

\section{Compliance}

Both participants were 100 percent compliant for clinic-PVR sessions. Home-PVR session compliance was based on information recorded by the MATLAB program. Participant 1 was 71 percent compliant during the first four weeks of the HEP and 10 percent during the last four weeks of the HEP. Participant 2 had a 64 percent compliance rate in his HEP.

\section{Upper Extremity Function and Symptoms}

Participant 1 exhibited mixed changes in UE functional ability.

\section{ARAT}

Participant 1 improved his right pinch and total score throughout the study. $\mathrm{He}$ also showed improvements in his left grasp, pinch, and total score at the midpoint. However, Participant 1 lost function on the left side at study conclusion and none of the changes reached the minimum clinically important difference (MCID) of 5.7 points [14].

\section{DASH}

Participant 1 had an increase in symptoms at week three, followed by improvements during weeks five and seven, and a slight increase in symptoms at week nine. Overall, Participant 1 surpassed the MCID of 10 points.

Table 2: Participant 1: Active Range of Motion Measured in Degrees

\begin{tabular}{|c|c|c|c|c|c|c|}
\hline & \multicolumn{3}{|c|}{ Left } & \multicolumn{3}{|c|}{ Right } \\
\hline & Week 1 & Week 5 & Week 9 & Week 1 & Week 5 & Week 9 \\
\hline Shoulder Flexion & 50 & 61 & 64 & 120 & 139 & 125 \\
\hline Shoulder Abduction & 70 & 81 & 57 & 125 & 130 & 115 \\
\hline Shoulder External Rotation & 35 & 40 & 30 & 60 & 70 & 57 \\
\hline Elbow Flexion & 120 & 120 & 121 & 135 & 143 & 136 \\
\hline Forearm Supination & 45 & 45 & 58 & 50 & 60 & 70 \\
\hline
\end{tabular}

Note: Bold = Increase. 


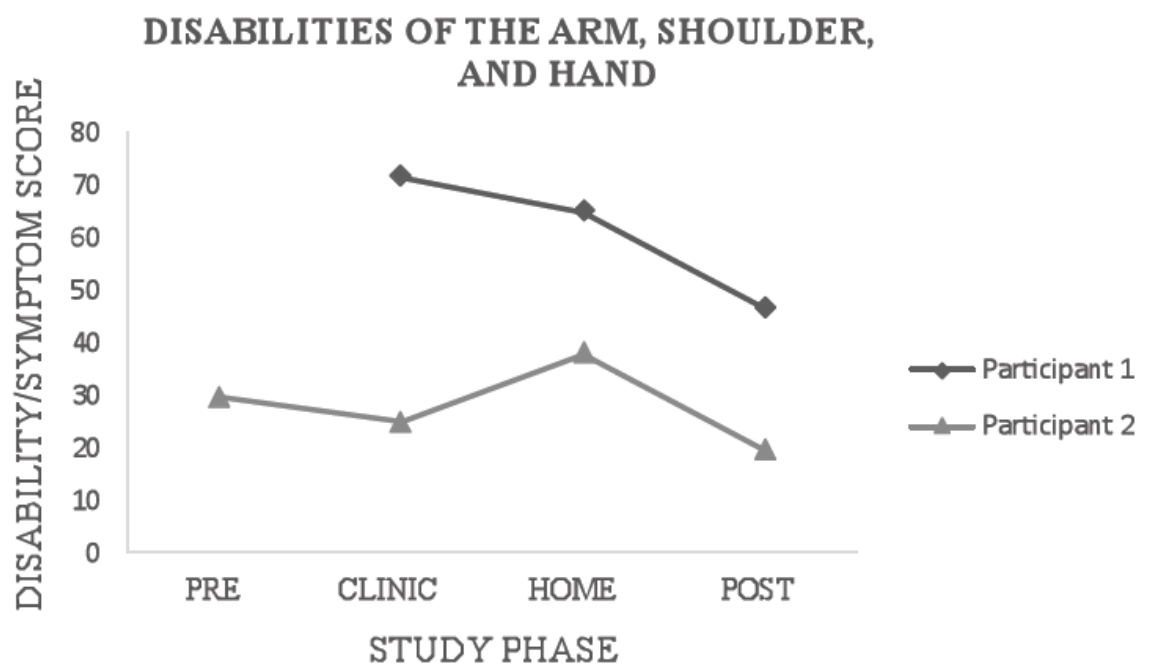

Figure 2: DASH total scores.

Note: Higher scores indicate more disability/symptoms. Study phases with multiple measurements were averaged.

ROM

Participant 1 also displayed mixed changes in AROM. He exhibited improved AROM in both shoulders, both forearm supinators, and right elbow flexion at the study's midpoint. At study conclusion, Participant 1 continued to show improvements in left shoulder flexion and both forearm supinators. However, he had decreased range in shoulder abduction, external rotation bilaterally, and right elbow flexion (Table 2).

Participant 2's UE functional ability also showed mixed results.

\section{ARAT}

Participant 2's score fluctuated around the average score of post-stoke patients (29.2) and never exceeded the MCID of 5.7 [14]. The therapist suggested that, because of his spasticity, the ARAT may not have been sensitive enough to measure the changes in ROM and compensations.

\section{DASH}

Participant 2 improved by 10-points on the DASH, meeting the MCID for the measure (Figure 2).

\section{ROM}

Participant 2's ROM measurements varied throughout the study. While several measurements showed improvement, the most prominent gains were in his active and passive ROM for external rotation, which was the primary focus of his games (Table 3 ). This increase in ROM occurred during the last two measurements while he utilized the system at home.

\section{Repetitions}

Participant 1 played approximately 11 hours of PVR games and completed about one repetition every 6.6 seconds. This resulted in an estimated 6,000 repetitions, 52 percent more than what is achieved during typical rehabilitation in the clinic [2]. Averaged between all his games, Participant 2 completed 1 repetition every 3.5 seconds for an estimated 515

Table 3: Participant 2: Active Range of Motion Measured in Degrees

\begin{tabular}{|c|c|c|c|}
\hline Right UE Movement & Pre-PVR & Home PVR & Post-PVR \\
\hline \hline Shoulder Flexion & 88 & $\mathbf{1 0 8}$ & 115 \\
\hline Shoulder Abduction & 110 & $\mathbf{1 1 7}$ & $\mathbf{8 7}$ \\
\hline Shoulder External Rotation & 38 & $\mathbf{8 5}$ & 132 \\
\hline Elbow Flexion & 132 & $\mathbf{1 3 5}$ & $\mathbf{7 8}$ \\
\hline Forearm Supination & 66 & & $\mathbf{8 3}$ \\
\hline
\end{tabular}

Note: $\mathrm{UE}=$ Upper Extremity; Bold $=$ Increase . 


\section{INTRINSIC MOTIVATION INVENTORY}

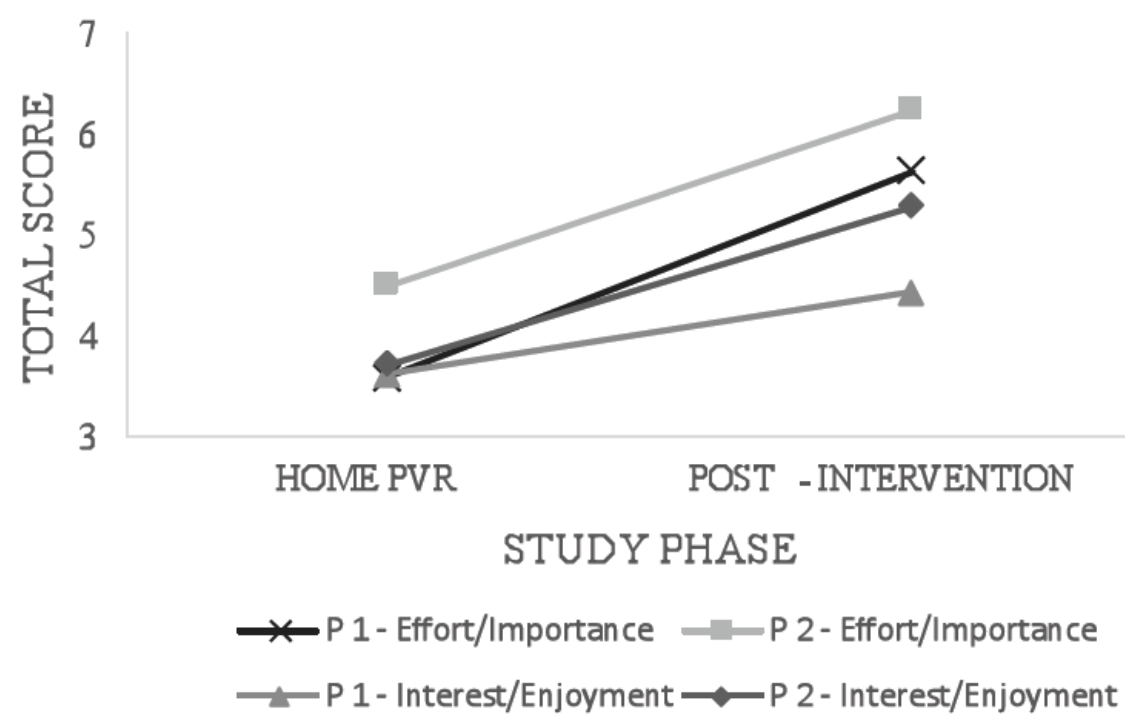

Figure 3: IMI total scores.

Note: $P=$ Participant number.

repetitions in each 40 minute PVR session. This means that Participant 2 generated around 4,630 repetitions during the home phase even with his moderate 64 percent HEP compliance.

\section{Motivation}

Participant 1's IMI interest/enjoyment scores improved 24 percent and the effort/importance scale improved 50 percent (Figure 3 ). He also rated the following statement "I believe doing this activity could be beneficial to me" a $7 / 7$ at the study's conclusion. The average positive comments on interest/enjoyment and effort/importance made by Participant 2 increased while the average negative comments decreased by the study's conclusion.

\section{Occupational Performance}

\section{COPM}

Participant 1 selected showering, toilet transfers, dressing, driving, and walking as the areas he would like to improve most. Between baseline and study conclusion, he showed MCID improvements in walking, toilet transfers, and showering performance and walking, driving, toilet transfers, and showering satisfaction. However, driving performance and dressing satisfaction and performance remained essentially the same (Table 4).

Participant 2 chose the pitching motion on the COPM because his primary recreational activity was softball. Given his spasticity and hypotonicity, the wind-

Table 4: COPM Scores: Participants' Self-Perception of Occupational Performance

\begin{tabular}{|c|c|c|c|c|c|}
\hline & & \multicolumn{2}{|c|}{ Baseline } & \multicolumn{2}{|c|}{ Post-Intervention } \\
\hline & & Performance & Satisfaction & Performance & Satisfaction \\
\hline \multirow[t]{6}{*}{ Participant 1} & Walking & 3 & 5 & 5 & 9 \\
\hline & Driving & 1 & 1 & 1 & 3 \\
\hline & Dressing & 8 & 7 & 7 & 8 \\
\hline & Toilet Transfer & 1 & 1 & 6 & 9 \\
\hline & Showering & 1 & 1 & 6 & 7 \\
\hline & Total & 2.8 & 3 & 5 & 7.2 \\
\hline Participant 2 & Right Arm Pitching & 4 & 4 & 8 & 8 \\
\hline
\end{tabular}

Note: Bold $=$ MCID 
up or external rotation was limited in strength and ROM. His overall scores doubled the MCID of the COPM for performance and satisfaction (Table 4).

\section{DISCUSSION}

The purpose of this pilot study was to test the feasibility and usability of PVR in the clinic and as a HEP. The secondary purpose was to evaluate the effects of PVR on UE function and occupational performance. Our hypothesis was that PVR would be feasible in the clinic and home and that using a motivating and engaging therapy experience would improve compliance, functional motor performance, and occupational performance.

The PVR system was successfully used in the rehabilitation clinic and home settings, though not without some complications. Further refinement would be necessary before the system could be used in the clinic without researcher support. Home-PVR had very few and only minor difficulties. A large scale research study could provide additional support to determine whether home-PVR could be used to increase motivation, repetitions, and ultimately, function and occupational performance.

Both participants achieved improvements reaching a MCID on the DASH and COPM. These results along with the interviews, show that improvements made using PVR have translated to functional tasks at home. Despite imperfect compliance, both participants' intrinsic motivation steadily increased throughout the study. This was likely attributable to the ability to change the games based on weekly feedback, improved game playing skills, and increased functional abilities. If motivation remained high, it is likely that compliance and functional abilities would have continued to improve also, furthering the cycle of motivation, compliance, and abilities.

\section{Clinical Significance}

HEPs are an important part the rehabilitation process. PVR allows therapists to match clients' interests to thousands of high-quality internet games, customize them to work with almost any movement, and increase the movement threshold for game activation. In addition, the low equipment cost makes it an affordable option for clinical and home use compared to previous lower-quality [19-21] and costly [11] virtual reality games. A clinic-to-home PVR approach allows therapists to observe progress in the clinic and make changes remotely. This ability to tailor targeted movements to novel games keeps clients motivated, engaged, and sufficiently challenged over a much longer period of time than traditional clinical interventions and HEPs. It also provides flexibility and support in their rehabilitation as the client's abilities and circumstances change. Such features may be useful as a transitionary component to a telerehabilitation program. Lastly, preliminary evidence suggests this protocol can improve motivation, compliance, and occupational performance. Scores on the COPM and DASH show a decrease in symptoms and an increase in ability to complete functional tasks. While the participants in this study had hemiplegia as a result of stroke, the customizability of the system allows for the therapist to adapt the program to fit the needs of other neuromuscular disabilities.

\section{Study Limitations}

The current feasibility study had several limitations. First, the use of two case studies prevents the results from being generalized to the stoke population as a whole. More participants would be required to continue to refine the protocol and evaluate its efficacy.

Second, Participant 1's comorbid medical conditions may have interfered with HEP compliance and therefore study results. Decreases in HEP compliance during the second half of the intervention were likely due to an acute exacerbation of renal failure due to missed dialysis appointments. Extending the intervention to promote recovery may have provided a more accurate representation of his compliance and improvements. Unfortunately, this was not feasible with study time constraints.

Third, although compliance for home-PVR was better than traditional HEPs, it still could have been improved. However, due to a large amount of data collected using MATLAB, the data were stored on the physical computer used by the participants for their HEP. These data were not available for verification until after the equipment was returned at the end of the study. Researchers had to rely on the participants' selfreports of home-PVR compliance during the study. A larger cloud-based data storage would have allowed researchers to check-in on participant HEP compliance and come up with additional strategies, if needed.

Fourth, caregiver assistance was needed to set-up the PVR system for Participant 1 to play games at home. This also may have affected compliance 
because help was not always available when he wanted to play. Further simplification of the game setup process is needed to allow more independence in playing the games.

Fifth, some technological challenges were experienced. Due to the large hospital setting, clinic computers were updated regularly, which removed the FAAST software and Kinect drivers from the computers, preventing use of PVR in the clinic. In addition, many administrative controls were in place that blocked use of gaming websites and required administrative rights to update the flash player software. Solving these challenges required a great deal of time and effort from administrative personal. Therefore, researcher laptops were used instead. However, this lead to its own complications with wireless network connectivity. Although the system was always at the mercy of the wireless connection, the problem could often be bypassed by preloading the games in a different location from the rehabilitation gym. Other technology challenges included the launch pad's limit of two movements for controlling games and difficulty finding games primarily controlled by two movements.

Finally, the PVR system could not be permanently set-up in the clinic due to limited space. This meant the equipment needed to be set-up and broken down before and after clinic-PVR sessions. This process would have used valuable therapy time. Therefore, researchers provided set-up for most clinic-PVR sessions. In addition, the lack of permanent PVR setup prevented the OT from gaining valuable practice and experience using the system. To ensure the participants had games that met their interests and had enough time to play in the clinic, researchers found and programmed the games according to the therapist's recommendations.

\section{CONCLUSIONS}

The current study has provided evidence to the feasibility and effectiveness of the PVR system. PVR is a promising low-cost therapeutic tool for improving compliance by incorporating the client's interests, abilities, and goals into a motivating and engaging intervention using internet games. However, further refinement and evaluation are needed to ultimately determine the feasibility and effectiveness without researcher assistance. Additional research should be conducted with larger sample sizes and with clients who have other movement disorders.

\section{REFERENCES}

[1] Mozaffarian D, Benjamin EJ, Go AS, Arnett DK, Blaha MJ, Cushman M, et al. Heart Disease and Stroke Statistics-2015 Update: A Report from the American Heart Association 2015; 131.

[2] Lang CE, MacDonald JR, Gnip C. Counting repetitions: an observational study of outpatient therapy for people with hemiparesis post-stroke. J Neurol Phys Ther 2007; 31: 3-10. http://dx.doi.org/10.1097/01.NPT.0000260568.31746.34

[3] Grindley EJ, Zizzi SJ. Using a Multidimensional Approach to Predict Motivation and Adherence to Rehabilitation in Older Adults. Top Geriatr Rehabil 2005; 21: 182. http://dx.doi.org/10.1097/00013614-200507000-00004

[4] Birkenmeier RL, Prager EM, Lang CE. Translating animal doses of task-specific training to people with chronic stroke in 1-hour therapy sessions: a proof-of-concept study. Neurorehabil Neural Repair 2010; 24: 620-35. http://dx.doi.org/10.1177/1545968310361957

[5] Yin CW, Sien NY, Ying LA, Chung SFCM, Leng DTM. Virtual reality for upper extremity rehabilitation in early stroke: A pilot randomized controlled trial. Clin Rehabil 2014; 28: 1066. http://dx.doi.org/10.1177/0269215514532851

[6] Holden MK. Virtual environments for motor rehabilitation: review. Cyberpsychol Behav 2005; 8: 187-211; discussion 212-9.

[7] Laver K, George S, Thomas S, Deutsch JE, Crotty M. Virtual reality for stroke rehabilitation. Stroke 2012; 43: 2012-4. http://dx.doi.org/10.1161/STROKEAHA.111.642439

[8] Saposnik G, Levin M. Virtual reality in stroke rehabilitation: A meta-analysis and implications for clinicians. Stroke 2011; 42: $1380-6$.

http://dx.doi.org/10.1161/STROKEAHA.110.605451

[9] Proffitt RM, Alankus G, Kelleher CL, Engsberg JR. Use of computer games as an intervention for stroke. Top Stroke Rehabil 2011; 18: 417-27. http://dx.doi.org/10.1310/tsr1804-417

[10] Lauterbach SA, Foreman MH, Engsberg JR. Computer games as therapy for persons with stroke. Games Health J 2013; 2: 24-8.

http://dx.doi.org/10.1089/g4h.2012.0032

[11] Lin J, Kelleher CL, Engsberg JR. Developing home-based virtual reality therapy interventions. Games Health J 2013; 2 : 34-8.

http://dx.doi.org/10.1089/g4h.2012.0033

[12] Gajdosik RL, Bohannon RW. Clinical measurement of range of motion. Review of goniometry emphasizing reliability and validity. Phys Ther 1987; 67: 1867-72.

[13] McAuley E, Duncan T, Tammen VV. Psychometric properties of the Intrinsic Motivation Inventory in a competitive sport setting: A confirmatory factor analysis. Res $Q$ Exerc Sport 1989; 60: 48-58.

http://dx.doi.org/10.1080/02701367.1989.10607413

[14] van der Lee JH, Beckerman H, Lankhorst GJ, Bouter LM. The responsiveness of the Action Research Arm Test and the Fugl-Meyer Assessment Scale in chronic stroke patients. J Rehabil Med 2001; 33: 110-3. http://dx.doi.org/10.1080/165019701750165916

[15] Beaton DE, Katz JN, Fossel AH, Wright JG, Tarasuk V Bombardier $C$. Measuring the wole or the parts? Validity, reliability, and responsiveness of the Disabilities of the Arm, Shoulder, and Hand Outcome Measure in different regions of the upper extremity. J Hand Ther 2001; 14: 128-46. http://dx.doi.org/10.1016/S0894-1130(01)80043-0

[16] Institute for Work \& Health. The DASH out come measure: disabilities of the arm, shoulder, and hand 2012. http: //www.dash.iwh.on.ca/home (accessed February 10, 2012). 
[17] Law M, Baptiste S, McColl M, Opzoomer A, Polatajko H, Pollock N. The Canadian Occupational Performance Measure: An outcome measure for occupational therapy. Can J Occup Ther 1990; 57: 82-7. http://dx.doi.org/10.1177/000841749005700207

[18] Law M, Baptiste S, Carswell A, McColl MA, Polatajko H, Pollock N. The Canadian Occupational Performance Measure. 2nd ed. Ottawa: CAOT Publications 1998.

[19] Proffitt R, Kelleher C, Baum C, Engsberg J. Using Alice 2.0 to design games for people with stroke. Games Health $\mathrm{J}$ 2012; 1: 303-7.

http://dx.doi.org/10.1089/g4h.2012.0029
[20] Kelleher C, Tam S, May M, Profitt R, Engsberg J. Towards a therapist-centered programming environment for creating rehabilitation games. Proc CGAMES'2011 USA - 16th Int Conf Comput Games Al, Animat Mobile, Interact Multimedia, Educ Serious Games 2011: 240-7. http://dx.doi.org/10.1109/cgames.2011.6000346

[21] Alankus G, Proffitt R, Kelleher C, Engsberg J. Stroke therapy through motion-based games. ACM Trans Access Comput 2011; 4: 1-35.

http://dx.doi.org/10.1145/2039339.2039342

Received on 14-07-2016

DOI: http://dx.doi.org/10.6000/2292-2598.2016.04.03.3

(c) 2016 Behar et al.; Licensee Lifescience Global.

This is an open access article licensed under the terms of the Creative Commons Attribution Non-Commercial License (http://creativecommons.org/licenses/by-nc/3.0/) which permits unrestricted, non-commercial use, distribution and reproduction in any medium, provided the work is properly cited. 